\title{
High-density SNP linkage map construction and QTL mapping for flavonoid-related traits in a tea plant (Camellia sinensis) using $2 b-R A D$ sequencing
}

\author{
$\mathrm{Li}^{-Y i} \mathrm{Xu}^{1,2+}$, Li-Yuan Wang ${ }^{1 *+}$, Kang Wei ${ }^{1}$, Li-Qiang Tan ${ }^{3}$, Jing-Jing Su ${ }^{1}$ and Hao Cheng ${ }^{1 *}$
}

\begin{abstract}
Background: Flavonoids are important components that confer upon tea plants a unique flavour and health functions. However, the traditional breeding method for selecting a cultivar with a high or unique flavonoid content is time consuming and labour intensive. High-density genetic map construction associated with quantitative trait locus (QTL) mapping provides an effective way to facilitate trait improvement in plant breeding. In this study, an F1 population ( $L \mathrm{~J} 43 \times \mathrm{BHZ}$ ) was genotyped using 2b-restriction site-associated DNA (2b-RAD) sequencing to obtain massive single nucleotide polymorphism (SNP) markers to construct a high-density genetic map for a tea plant. Furthermore, QTLs related to flavonoids were identified using our new genetic map.

Results: A total of 13,446 polymorphic SNP markers were developed using 2b-RAD sequencing, and 4,463 of these markers were available for constructing the genetic linkage map. A 1,678.52-cM high-density map at an average interval of $0.40 \mathrm{cM}$ with 4,217 markers, including 427 frameset simple sequence repeats (SSRs) and 3,800 novel SNPs, mapped into 15 linkage groups was successfully constructed. After QTL analysis, a total of 27 QTLs related to flavonoids or caffeine content (CAF) were mapped to 8 different linkage groups, LG01, LG03, LG06, LG08, LG10, LG11, LG12, and LG13, with an LOD from 3.14 to 39.54 , constituting $7.5 \%$ to $42.8 \%$ of the phenotypic variation.

Conclusions: To our knowledge, the highest density genetic map ever reported was constructed since the largest mapping population of tea plants was adopted in present study. Moreover, novel QTLs related to flavonoids and CAF were identified based on the new high-density genetic map. In addition, two markers were located in candidate genes that may be involved in flavonoid metabolism. The present study provides valuable information for gene discovery, marker-assisted selection breeding and map-based cloning for functional genes that are related to flavonoid content in tea plants.
\end{abstract}

Keywords: Genetic map, SNP, QTL mapping, 2b-RAD sequencing

\section{Background}

Tea plants [Camellia sinensis (L.) Kuntze] are the raw materials for one of the three most popular non-alcoholic drinks in the world [1,2]. Because of its high economic value, it has been extensively planted in China, India, Sri Lanka and so on [3, 4]. Therefore, selecting new tea cultivars with improved production

\footnotetext{
* Correspondence: wangly@tricaas.com; chenghao@tricaas.com

${ }^{+} \mathrm{Li}-\mathrm{Yi} \mathrm{Xu}$ and Li-Yuan Wang contributed equally to this work.

${ }^{1}$ National Centre for Tea Improvement, Tea Research Institute, Chinese

Academy of Agricultural Sciences, Hangzhou 310008, China

Full list of author information is available at the end of the article
}

and quality characteristics has received much attention in these countries. However, compared with other crops, tea plants have a high self-incompatibility and require more than three years to grow and develop, which makes it difficult to carry out genetic improvement [5], while traditional breeding methods are still commonly used. A genetic linkage map, particularly a high-density map, plays a major role in plant breeding as a fundamental tool for genetic analysis and further functional gene localization. Such maps are based on calculating the recombination rates between many molecular

(c) The Author(s). 2018 Open Access This article is distributed under the terms of the Creative Commons Attribution 4.0 International License (http://creativecommons.org/licenses/by/4.0/), which permits unrestricted use, distribution, and reproduction in any medium, provided you give appropriate credit to the original author(s) and the source, provide a link to the Creative Commons license, and indicate if changes were made. The Creative Commons Public Domain Dedication waiver (http://creativecommons.org/publicdomain/zero/1.0/) applies to the data made available in this article, unless otherwise stated. 
markers [6-8]. Thus, constructing genetic maps and further quantitative trait locus (QTL) analysis should be effective methods to facilitate trait improvement in breeding tea cultivars.

With advances in DNA sequencing technology, molecular marker technologies, especially SNPs (single nucleotide polymorphisms), play a major role in breeding [9-11]. Traditionally, randomly amplified polymorphic DNA (RAPD), amplified fragment length polymorphism (AFLP), restriction fragment length polymorphism (RFLP) and simple sequence repeats (SSRs) have been used to construct genetic maps and identify QTLs in plants [12, 13]. However, they have a low density and uneven distribution throughout the genome, which restricts the accuracy of QTL analysis [14]. SNP markers based on high-throughput sequencing for whole or partial genomes have a high density and even distribution in genomes, and they have been rapidly developed as new molecular markers [15]. 2b-Restriction site-associated DNA (2bRAD) sequencing is a high-throughput sequencing method that uses type IIB restriction endonucleases to produce uniform fragments for sequencing. Benefiting from the character of type IIB restriction endonucleases, these fragments are of the same length and have high coverage in chromosomes, which make them particularly suitable for high-throughput genotyping to construct genetic linkage maps in natural populations [16]. Therefore, in this study, 2b-RAD sequencing was chosen to develop SNP markers to construct a high-density genetic map for a tea plant.

Based on genotyping for molecular markers, some genetic maps have recently been constructed by using various types of markers in tea plants. For example, Chang et al. [17] constructed a genetic map using 678 markers including 143 RAPDs, 11 public SSRs, 495 AFLPs, and 29 newly mined SSRs, and the map covered $1441.6 \mathrm{cM}$ with an average distance of $4.7 \mathrm{cM}$ between adjacent markers. Ma et al. [18] developed an SSR genetic map containing 406 SSRs spanning $1143.5 \mathrm{cM}$ with an average distance of $2.9 \mathrm{cM}$ between markers. Then, they re-constructed a SNP/SSR genetic map with 6,042 SNPs and 406 SSRs distributed over 3,965 cM with an average distance of $1.0 \mathrm{cM}$ between markers using specific length amplified fragment (SLAF)-seq [19]. However, to our knowledge, the average distance between markers is still more than $1.0 \mathrm{cM}$ in all published genetic maps for tea plants. Thus, we attempted to construct a denser genetic map by using large SNP markers based on $2 \mathrm{~b}-\mathrm{RAD}$ sequencing to improve the precision of QTL analysis and reduce the difficulty of fine QTL mapping.

In general, major secondary metabolites containing flavonoids, caffeine and amino acids are important components of tea extraction that provide the rich flavours, fresh taste and nutrient content of tea products [20].
Among them, flavonoids are the most abundant component, are driven by phenylalanine and include flavones, flavonols, isoflavones, flavanones, flavanols, and anthocyanidins [21]. In flavonoids, catechin components are the most prevalent, and their ability to effectively react with oxygen has proven them to be scavengers [22]. The variation in catechins in young leaves is responsible for the various quality and flavour characteristics during tea production. The lower levels of catechins can make green tea to taste less astringent and more umami [23, 24]. In addition, flavonoids with a wide range of functions, for example, antioxidant activity, ultraviolet light protection, and defence against phytopathogens, were also found [25]. However, only one QTL region related to the catechin content, timing of spring bud flush, young shoot colour (YSC) and mature leaf size has been identified in the SSR genetic map $[18,26]$. Due to a small mapping population or few markers in the genetic map, some maps may be unsuitable for QTL mapping. Thus, it is necessary to construct a high-density SNP genetic linkage map and identify the QTLs with flavonoid-related traits for further functional gene discovery and molecular marker-assisted breeding of tea plants.

In this study, the aims were 1) to construct a high-density genetic map using $2 \mathrm{~b}-\mathrm{RAD}$ sequencing, 2) to map QTLs for flavonoid-related traits in the F1 group, and 3) to select candidate genes that may affect the target trait via sequence comparison and functional annotation.

\section{Methods}

Plant materials and DNA extraction

The F1 segregating population consisted of 327 individuals was derived from an inter-varietal cross between two clonal tea cultivars, C. sinensis cv. Longjing 43 ('LJ43') and C. sinensis cv. Baihaozao ('BHZ'). 'LJ43' was used as the female parent. All individuals were planted at the germplasm resources of the Tea Research Institute in the Chinese Academy of Agricultural Sciences in Hangzhou, Zhejiang, China. Detailed information on the F1 population was published in Tan et al. [8]. Fresh and tender leaves from each of the 327 progenies and their parents were collected to extract genomic DNA using a Tiangen Plant DNA Kit DP305 (Beijing, China). The quality and concentration of the extract products were detected using a Nanodrop ND-2000 (Thermo Scientific, USA), and they were preserved at $-20^{\circ} \mathrm{C}$.

\section{Library preparation, sequencing and de novo genotyping}

The genomic DNA of the 327 progenies and two parents were used to construct $2 \mathrm{~b}$-Rad libraries using type IIB restriction enzymes, following the protocol developed by Wang et al. [16]. First, 100-200 ng of genomic DNA was 
digested at $37^{\circ} \mathrm{C}$ for $3 \mathrm{~h}$ in a $15-\mu \mathrm{l}$ reaction that contained 4. $\mathrm{U}$ of BsaXI. Then, the digestion product was incubated at $4^{\circ} \mathrm{C}$ for $16 \mathrm{~h}$ in a $12-\mu$ ligation master mix consisting of $0.2 \mu \mathrm{M}$ library-specific adaptors, $1 \mathrm{mM}$ ATP and $800 \mathrm{U}$ of T4 DNA ligase. In detail, after type IIB restriction enzyme (BsaXI) digesting, 33bp tags (5'-NNNNNNNNNACNN NNNCTCCNNNNNNNNNn-3') were obtained. Among the tag, 'NNn' of 3' sticky end could be paired with special adaptors consisted of 6 bp recognition base and 3 bp overhang (Additional file 1: Table S1). With changing of base, various overhang-types were formed, for instance, the 5'-NNN-3' overhang containing a total of 64 overhangtypes while only 16 overhang-types in $5^{\prime}$-NNA-3' or 5 '-NNT-3' overhang-type. We could change the last base of overhang to reduce the density of tags to save cost. In our study, adaptors with 5'-NNN-3' overhang were used to construct the standard BsaXI libraries of two parents, whereas adaptors with 5'-NNA-3' and 5'-NNT-3' overhangs were constructed for each progeny to reduce the representation library. In the second PCR amplification, a specific adapter barcode was incorporated into each library; then, all libraries were pooled for single-end sequencing $(1 \times 50 \mathrm{bp})$ using an Illumina HiSeq 2000 sequencer.

De novo genotyping followed the analytical approach described by Jiao et al. [27]. Briefly, reads with the following characteristics were removed: no restriction sites, long homopolymers (more than $10 \mathrm{bp}$ ) and low quality (more than 10 bases with a quality fraction of $<20$ ). RADtyping program v. 1.3, which is an integrated package for accurate de novo codominant and dominant RAD genotyping in mapping populations, was used for the de novo $2 \mathrm{~b}-\mathrm{RAD}$ genotyping using the default parameters [28].

\section{2b-RAD data analysis}

The markers were genotyped according to the RAD-typing approach for the non-reference genomes [29]. In general, the markers of the two parents were used to construct a reference sequence. Then, the high-quality reads of the progenies were mapped to a high-quality reference sequence and clustered to obtain unique tags for genotyping by SOAP software (http:// soap.genomics.org.cn) [29]. Unique markers that had a sequencing depth of less than 3 were removed.

\section{Genetic linkage mapping}

Markers that could be genotyped in at least $80 \%$ of the progenies were chosen for further analysis. The genetic maps were constructed by high-quality SNP markers that conformed to the Mendelian separation ratio $(\mathrm{p} \geq$ 0.05 ) via the $x 2$ test and a set of 483 SSR markers previously mapped in this population [30].
First, sex-specific maps were constructed for each parent using the two-way pseudo-testcross strategy [31]. Female and male datasets were created using the command 'create maternal and paternal population nodes' in the JoinMap 4.0 program [32]. The heterozygous markers in either the female or the male were tagged as ' $\mathrm{f}$ ' of ' $\mathrm{m}$ ', and bi-markers were tagged as ' $\mathrm{h}$ ', whereas the dominant markers were tagged as ' $\mathrm{df}$ ' or 'dm'. Markers were grouped at a logarithm of odds (LOD) threshold value of 5.0. The regression mapping algorithm was used for map construction. Marker distances in cM were calculated using Kosambi's mapping function. Linkage groups were drawn visually using the MapChart software [33]. A combined map was estimated by integrating the female and male maps using the shared markers in MergeMap software [34]. Two total genetic map lengths were estimated based on the combined map, Ge1 [35] and Ge2 [36], and their average was used as the predicted total genetic map length $(\mathrm{Ge})$. The genome coverage (Cof) was calculated by Gof/Ge, where Gof was the observed genetic map length (the sum of the map lengths of all linkage groups).

\section{Phenotype data collection}

Data for ten traits were collected for the QTL analysis, which contained the anthocyanin content (OPC), YSC, caffeine content (CAF) and seven components of catechins (GC, EGC, C, EC, EGCG, GCG and ECG). The YSC data came from our previous studies [30]. In this study, the CAF and the seven components of catechins in each mapping individual were measured using HPLC in 2014 and 2015, respectively. This spectrophotometric method was used to measure the OPC, which included the following steps: first, young shoots of each mapping individual were picked and freeze dried. Then, the OPC was extracted from the tea sample via incubation in a $0.1 \mathrm{~mol} / \mathrm{L}$ hydrochloric acid ethanol solution at $60^{\circ} \mathrm{C}$ for $1 \mathrm{~h}$. Finally, optical density values at $530 \mathrm{~nm}, 620 \mathrm{~nm}$ and $650 \mathrm{~nm}$ were measured to calculate the OPC. Repeat the process for each test and average the results.

\section{QTL mapping analysis}

QTL mapping analysis was performed for ten traits based on the combined linkage map in MapQTL 5 software [37]. In general, the LOD scores were analysed using the interval mapping method; then, the genome-wide and chromosome-wide LOD significance thresholds at the 95\% level were determined via a 1,000-permutation test for each trait. QTLs with LOD scores greater than the chromosome threshold at 95\% were declared significant. Finally, all QTLs were named according to Cui et al. [38]. 


\section{SNP annotation}

The mapped markers were aligned against the reference genome [39] using SOAP software. The parameters were set to (-r 0, -M 0), '-r 0' meaning no repeats and '-M 0' meaning no mismatch. After removing the unaligned marker, the markers that were mapped in the QTL regions were annotated to ensure the position and function in the reference genome using SnpEff 4.1 software.

\section{Results}

\section{2b-RAD library}

The 2b-RAD library of the 327 individuals and two parents generated a total of 1,002,035,680 reads (Table 1 ). The average number of reads of the standard library consisting of two parents was 40,833,712, whereas that of each progeny was 2,814,582, which used selective overhangs (5'-NNA-3' and 5'-NNT-3'). After sequence filtering and removing the low-quality reads, more than $85 \%$ of the 317 progeny libraries contained high-quality reads with a restriction site (Additional file 1: Table S1). After mapping the high-quality reads of the progenies into reference sequences, each progeny obtained 71,852 unique markers, and the sequencing depth reached an average of 20x, whereas there were 190,468 unique markers for each parent in which the sequencing depth reached an average of 123x (Additional file 2: Table S2).

\section{SNP markers}

A total of 46,932 2b-RAD loci with different genotypes in the two parents were detected (Additional file 3: Table S3). After filtering, both had low- or high-coverage locus clusters due to sequencing errors or repeat sequences, respectively, and SNP genotyping was performed. A total of 10,816 co-dominant and 2,630 dominant 2b-RAD markers were detected (Additional file 4: Table S4). Among these markers, three segregation types were classified, including $h k \times h k, l m \times l l$ and $n n \times n p$ (Fig. 1). Moreover, 2b-RAD markers with genotyping information in more than $80 \%$ of $\mathrm{F} 1$ individuals were selected. After the X2 test, a total of 3,026 co-dominant and 1,437 dominant 2b-RAD markers were available to construct the genetic linkage map. Among these markers, $77.7 \%$ were heterozygous in the female parent, whereas $74.0 \%$ were heterozygous in the male parent.

Table 1 The summaries of high-throughput sequencing

\begin{tabular}{llll}
\hline Sample & Original reads & High-quality reads & Ratio (\%) \\
\hline LJ43 & $44,091,387$ & $40,487,516$ & 91.8 \\
BHZ & $37,576,037$ & $33,514,594$ & 89.2 \\
327 Progenies & $920,368,256$ & $797,734,136$ & 86.7 \\
Total & $1,002,035,680$ & $871,736,246$ & 87.0 \\
\hline
\end{tabular}

\section{Genetic linkage map}

In this study, a total of 4,943 markers were used to construct the genetic linkage map, which consisted of 4,463 newly developed SNPs and 483 published SSRs. The SSR markers could be used to ensure the frame of the genetic linkage map. After genetic mapping using JoinMap 4.0, for both the female and male genetic maps, the markers were divided into fifteen linkage groups at a LOD threshold of 5.0. In the female genetic map, the linkage length ranged from 73.49 to $144.17 \mathrm{cM}$ with 82 to 290 markers. In total, the female map included 2,470 markers spanning $1427.70 \mathrm{cM}$ with an average interval of $0.58 \mathrm{cM}$. In the male genetic map, the linkage length ranged from 78.71 to $161.20 \mathrm{cM}$ with 76 to 208 markers. In total, the male map included 2,217 markers spanning $1710.63 \mathrm{cM}$ with an average interval of $0.78 \mathrm{cM}$ (Additional file 5: Table S5).

Furthermore, by integrating the female and male maps using the shared markers, a high-density map with 4,217 markers mapped into 15 linkage groups, including 427 SSRs and 3,800 SNPs, was successfully constructed (Table 2, Additional file 6: Figure S1). The combined map spanned a total of $1,678.52 \mathrm{cM}$ with an average marker distance of $0.40 \mathrm{cM}$ and an average of 281 markers in each linkage group (Additional file 7: Table S8). The genetic lengths of the 15 linkage groups ranged from 87.46 to $146.93 \mathrm{cM}$, with an average marker distance of 0.30 to $0.48 \mathrm{cM}$ in the fifteen linkage groups. The longest and largest linkage group was LG03, with a genetic distance of $146.93 \mathrm{cM}$ and 374 markers, whereas the shortest was LG15, which spanned $87.46 \mathrm{cM}$ and contained 223 markers. The smallest group was LG12, with 206 markers. The integrity of the combined map with gaps of less than $2 \mathrm{cM}$ between each adjacent marker ranged from 95.71\% (LG04) to 98.92\% (LG03), with an average of $98.19 \%$. The integrity of the 14 linkage groups was greater than 98\%, except for that of LG04, indicating the comparatively high coverage and integrity in these combined maps.

Moreover, to compare the map alignment and ensure the map orientation, the 427 shared SSR markers on our map were used to align the SSR-based genetic map constructed in our previous study [30]. Overall, we successfully aligned our genetic linkage map (Map1) with the SSR-based genetic map (Map2) at a one-to-one correspondence (Additional file 8: Figure S2). A relatively consistent order was observed for almost all linked markers between the two maps. However, only 3 markers changed over the long distance between linked markers in LG03, LG08 and LG10.

\section{Phenotypic statistical analysis of flavonoid-related traits} The variance degrees of the aimed traits among the segregating population and parents was an important 


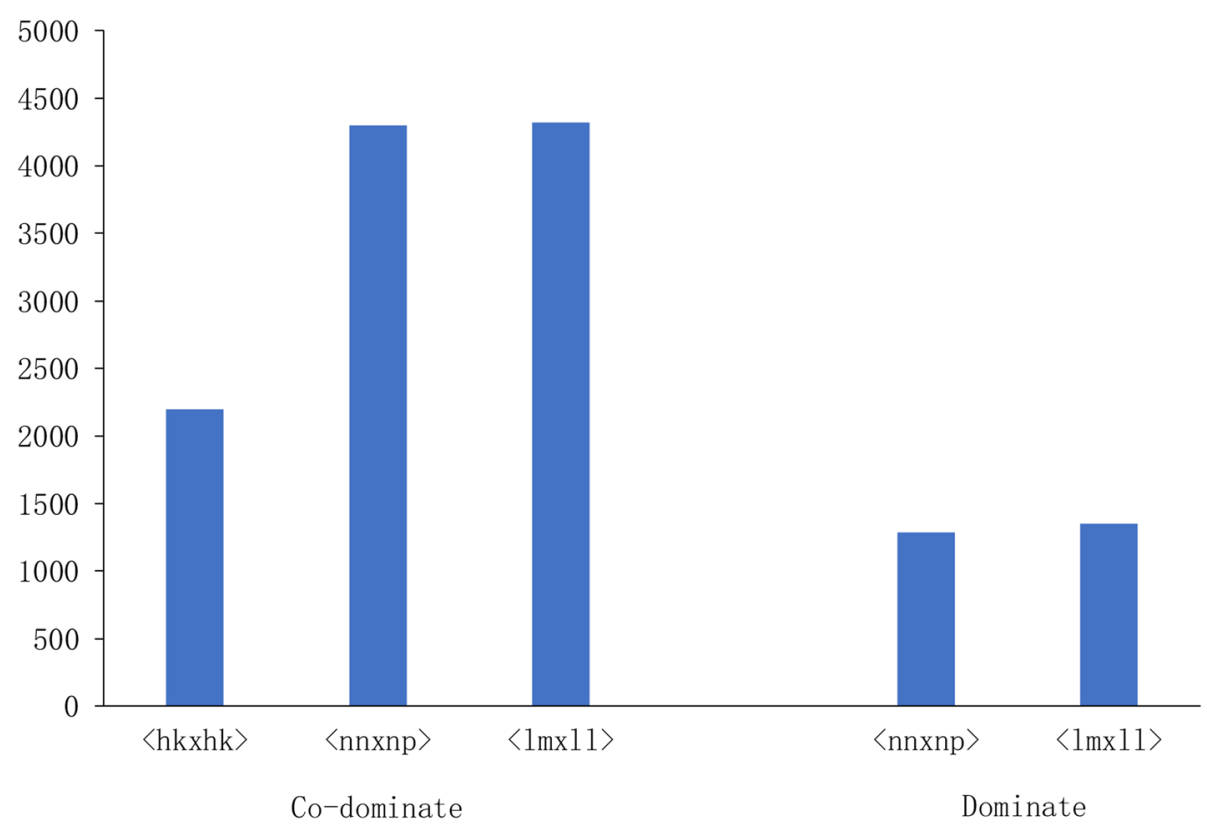

Segregation type

Fig. 1 Number of SNPs for segregation type (Co-dominate markers consists of three segregation types: hkxhk, Imxll, nnxnp; dominate markers consists of two segregation types: Imxll, nnxnp)

parameter for QTL mapping. Thus, values of coefficient of variation (C.V) were focused on afterward statistical analyses. The results showed the C.V of 9 traits ranged from $10.42 \%$ to $50.38 \%$ and almost all of them were above $20 \%$ (Table 3). So, there was a highly dispersed

Table 2 Information on the genetic map

\begin{tabular}{|c|c|c|c|c|c|c|c|}
\hline \multirow{2}{*}{$\begin{array}{l}\text { Linkage } \\
\text { group }\end{array}$} & \multicolumn{3}{|c|}{ Markers no. } & \multirow{2}{*}{$\begin{array}{l}\text { Distance }{ }^{a} \\
\text { (cM) }\end{array}$} & \multirow{2}{*}{$\begin{array}{l}\text { Length } \\
\text { (cM) }\end{array}$} & \multirow{2}{*}{$\begin{array}{l}\text { Gaps }<2 \text { cM } \\
(\%)\end{array}$} & \multirow{2}{*}{$\begin{array}{l}\text { Max Gap } \\
\text { (cM) }\end{array}$} \\
\hline & SSR & SNP & Total & & & & \\
\hline LG01 & 40 & 331 & 371 & 0.40 & 146.61 & 97.83 & 5.10 \\
\hline LG02 & 33 & 248 & 281 & 0.44 & 123.96 & 98.21 & 5.40 \\
\hline LG03 & 38 & 336 & 374 & 0.39 & 146.93 & 98.92 & 3.79 \\
\hline LG04 & 8 & 226 & 234 & 0.48 & 111.71 & 95.71 & 5.75 \\
\hline LG05 & 16 & 325 & 341 & 0.30 & 103.51 & 98.24 & 6.11 \\
\hline LG06 & 31 & 276 & 307 & 0.40 & 122.31 & 98.04 & 6.03 \\
\hline LG07 & 29 & 232 & 261 & 0.40 & 103.45 & 98.08 & 8.70 \\
\hline LG08 & 42 & 265 & 307 & 0.41 & 125.03 & 98.04 & 4.57 \\
\hline LG09 & 34 & 310 & 344 & 0.37 & 126.89 & 98.83 & 3.42 \\
\hline LG10 & 27 & 242 & 269 & 0.37 & 99.58 & 98.51 & 2.58 \\
\hline LG11 & 30 & 225 & 255 & 0.39 & 98.46 & 98.03 & 5.33 \\
\hline LG12 & 23 & 183 & 206 & 0.45 & 92.57 & 98.54 & 6.99 \\
\hline LG13 & 20 & 201 & 221 & 0.44 & 96.74 & 98.64 & 8.89 \\
\hline LG14 & 27 & 196 & 223 & 0.42 & 93.31 & 98.65 & 4.87 \\
\hline LG15 & 17 & 206 & 223 & 0.39 & 87.46 & 98.65 & 2.96 \\
\hline Total & 417 & 3800 & 4217 & 0.40 & 1678.52 & 98.19 & 8.89 \\
\hline
\end{tabular}

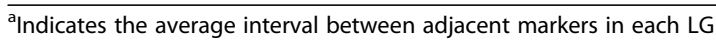

and variance degrees of traits among the segregating F1 population. Interestingly, we could find the values of C.V showed a substantial reduction from 2014 to 2015 year in each catechin components and CAF, which meant it existed deep environmental impact for different years. Even so, the distribution of F1 progenies was still discrete in each trait, but the range of variation was smaller (Fig. 2). Moreover, the Fig. 2 also displayed the great variation between two parents in flavonoid concentration and CAF.

\section{QTL mapping}

In our study, regions with LOD $\geq 3$ for each LG were identified as major QTLs. Thus, a total of 27 QTLs associated with ten traits were mapped to eight different linkage groups: LG01, LG03, LG06, LG08, LG10, LG11, LG12, and LG13 (Table 4, Additional file 9: Figure S3). Among these QTLs, 4 for OPC were identified on LG03 and LG08, with an $\mathrm{R}^{2}$ ranging from $20.3 \%$ (qOPC03a) to $42.0 \%$ (qOPC08a). Interestingly, 3 QTLs for YSC that were closely connected to the 4 QTLs for OPC were also mapped to LG03 and LG08. Additionally, the same closely related marker (f707) was found at the peak of qOPC08a and qYSC08a. It also increased at the peak of qOPC03a and qYSC03a (marker dm58). Moreover, three QTLs of OPC (qOPC08a, qOPC08b, qOPC03a) had an overlapping region with those of YSC (qYSC08a, qYSC08b, qYSC03a) on the genetic map. Among these QTLs, 2 of YSC were reported in previous studies, and 1 

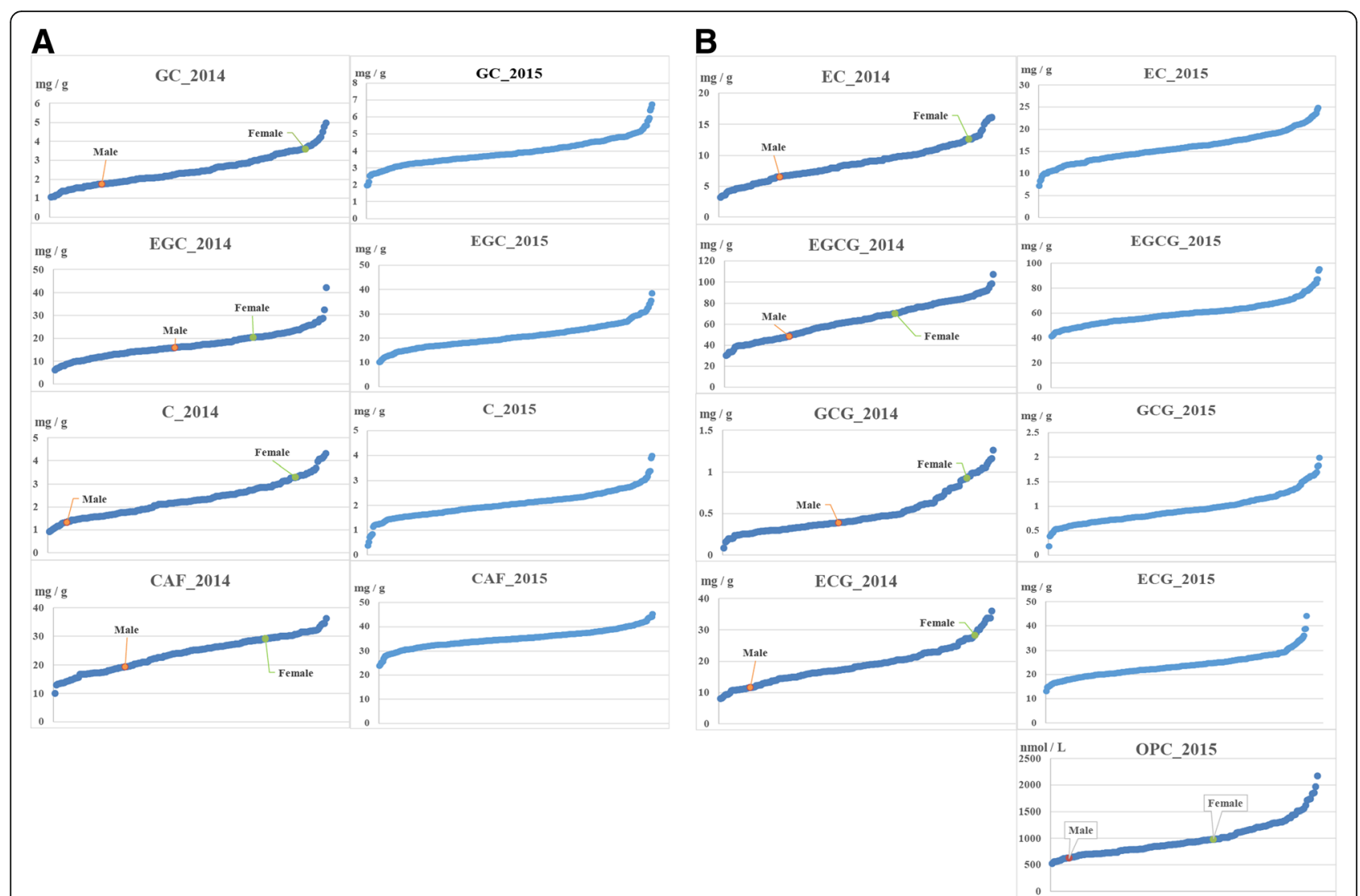

Fig. 2 The phenotypic distribution of flavonoid-related traits ( $X$ axis is 327 progenies and 2 parents)

new QTL (qYSC08b) was identified for the first time in this study.

Both two QTLs for CAF were identified on LG11, but they were in different positions in 2014 and 2015. The major QTL, qCAF11a, was located at $91.90 \mathrm{cM}$ with the largest $R^{2}(15.7 \%)$, and the marker $d m 870$ was close to it. The other two QTLs with a small $\mathrm{R}^{2}$ were located at $35.34 \mathrm{cM}(9.2 \%)$.

A total of 17 QTLs associated with seven components of catechins were mapped to 7 different linkage groups containing LG01, LG03, LG06, LG10, LG11, LG12, and LG13. Among these QTLs, 6 major QTLs of 4 components (qEGC06a, qEC06a, qEGCG06a, qEGCG06b, qECG06a, qECG06b) were primarily clustered into regions from $40 \mathrm{cM}$ to $60 \mathrm{cM}$ on LG06. The LOD of each QTL was relatively higher and ranged from 3.14 to 10.56. Remarkably, qEGCG06a and qEGCG06b were stable, and the major QTLs found via 2-year testing, except for qECG06a and qECG06b, were very close, although they had no overlaps. Another QTL cluster was in the region from 35 to $60 \mathrm{cM}$ in LG10. The QTLs of the $\mathrm{GC}$ were primarily clustered on this region, except for qGC01a, with an $\mathrm{R}^{2}$ from 9.4 to 10.5 , and one stable QTL of EGC (qEGC10a) was also identified in this region.
Two stable QTLs (qC12a, qECG12a) were distributed in different regions in LG12, with an $\mathrm{R}^{2}$ from 8.3 to 15.2 . Moreover, the other stable QTLs were distributed in LG01, LG03, LG07, LG11, and LG13; the number of QTLs in each LG varied from one to two; and the $\mathrm{R}^{2}$ values for these QTLs ranged from 7.7 (qEGCG01a) to 18.9 (qGCG11a).

\section{Marker annotation and candidate genes}

A total of 878 mapped markers were successful located in the reference genome, and 634 of them were located to the annotated regions (Additional file 10: Table S6, Additional file 11: Figure S4). Furthermore, based on QTL mapping, 86 markers were found in the QTL regions of ten flavonoid-related traits. To obtain a deep understanding of which markers in the QTL regions were related to flavonoids, we performed functional annotation and found that 64 QTLs had obtained annotation information. Of note, the gene functions that corresponded to 2 markers were considered related to the QTL (Table 5, Additional file 12: Table S7). Marker f707 at the peak LOD value for qOPC08a and qYSC08a has a basic helix-loop-helix domain, which may be a part of MYB-bHLH-WD40. MYB-bHLH-WD40 was confirmed to 
Table 3 The statistical analysis of phenotypic data

\begin{tabular}{llllllll}
\hline Trait $^{a}$ & Min & Max & Range & Mean & Std.E & Std.D & C.V (\%) \\
\hline OPC_2015 & 515.95 & 2169.53 & 1653.58 & 980.20 & 24.79 & 312.54 & 31.89 \\
GC_2014 & 1.05 & 4.98 & 3.92 & 2.47 & 0.06 & 0.80 & 32.30 \\
GC_2015 & 1.97 & 6.75 & 4.78 & 3.91 & 0.04 & 0.74 & 18.94 \\
C_2014 & 0.92 & 4.32 & 3.4 & 2.30 & 0.06 & 0.73 & 31.85 \\
C_2015 & 0.38 & 3.98 & 3.6 & 2.05 & 0.03 & 0.51 & 24.66 \\
EGC_2014 & 6.12 & 42.09 & 35.97 & 17.10 & 0.41 & 5.49 & 32.09 \\
EGC_2015 & 10.09 & 38.46 & 28.37 & 20.63 & 0.27 & 4.84 & 23.44 \\
CAF_2014 & 10.03 & 36.23 & 26.19 & 24.05 & 0.43 & 5.70 & 23.71 \\
CAF_2015 & 23.82 & 45.29 & 21.47 & 35.10 & 0.20 & 3.66 & 10.42 \\
EC_2014 & 3.18 & 16.09 & 12.91 & 8.70 & 0.21 & 2.81 & 32.33 \\
EC_2015 & 7.2 & 24.83 & 17.63 & 15.85 & 0.18 & 3.24 & 20.46 \\
EGCG_2014 & 30.09 & 107 & 76.91 & 63.81 & 1.26 & 16.70 & 26.17 \\
EGCG_2015 & 40.95 & 95.04 & 54.09 & 59.81 & 0.52 & 9.24 & 15.45 \\
GCG_2014 & 0.08 & 1.26 & 1.18 & 0.49 & 0.02 & 0.25 & 50.38 \\
GCG_2015 & 0.18 & 1.98 & 1.81 & 0.94 & 0.02 & 0.29 & 30.95 \\
ECG_2014 & 8.08 & 36 & 27.92 & 18.73 & 0.44 & 5.82 & 31.07 \\
ECG_2015 & 13.15 & 44.05 & 30.9 & 23.61 & 0.26 & 4.57 & 19.35 \\
\hline aTh
\end{tabular}

The unit of OPC_2015 is $\mathrm{nmol} / \mathrm{L}$, and other traits are all $\mathrm{mg} / \mathrm{g}$

regulate anthocyanin biosynthesis in plants [40]. Marker f252 in qECG12a has been annotated as chalconeflavanone isomerase, which is an essential enzyme of the flavonoid synthesis pathway [41, 42].

\section{Discussion}

\section{2b-RAD is a suitable method for genotyping}

For SNP genotyping, among sequencing technologies with sophisticated tools, such as Stacks, RAD is the most widely applied method to date [43]. However, it is difficult to make a parallel for high-throughput sample processing due to the labour-intensive and time-consuming nature of creating RAD libraries [44]. A notable feature of $2 \mathrm{~b}-\mathrm{RAD}$ sequencing is the tunable genome representation from libraries by using less degenerate adaptors, such as 5'-NNA-3' overhangs, which can significantly reduce the cost and workload with an acceptable accuracy [45]. Thus, in contrast to the traditional genotyping method, 2b-RAD is more cost effective and flexible and has been successfully used for developing SNP markers, genotyping and constructing genetic maps. For instance, Cui et al. presented a high-density linkage map that covers $98.5 \%$ of the genome with 73 linkage groups using $2 \mathrm{~b}-\mathrm{RAD}$, and they revealed a ZW-ZZ sex determination system in Eriocheir sinensis based on QTL analysis of the gender phenotype [46].

In our research, a large number of SNP markers were successful discovered and genotyped in the F1 population of 'LJ $43 \times \mathrm{BHZ}$ ' using $2 \mathrm{~b}-\mathrm{RAD}$. Using high-throughput sequencing and genotyping, a total of 46,932 2b-RAD markers were developed, and of these, 10,816 co-dominant and 2,630 dominant 2b-RAD markers were polymorphic. Among these markers, 4463 markers that passed the $\mathrm{x} 2$ test were selected to construct a genetic linkage map. Due to massive sequencing and strict filtration, the marker quality and quantity conformed to the requirements for constructing a genetic map. Therefore, our study further indicates that $2 \mathrm{~b}-\mathrm{RAD}$ is a suitable method for genotyping tea plants.

\section{Mapping strategy and population construction}

The high self-incompatibility of tea plants [5] it makes it difficult to construct a common population for genotyping and QTL analysis, such as an F2 population, RIL population, and NIL population. Thus, we chose an alternative method to construct an F1 population for genotyping based on a 'pseudo-testcross' mapping strategy. This strategy considers that many loci in the cross between two heterozygous individuals will be heterozygous in one parent and null in the other and will segregate 1:1 in their F1 progeny following a testcross configuration [31]. Therefore, the gametic segregation in each individual can be directly and efficiently analysed, and this strategy has been widely applied to construct an F1 population in woody perennials [47]. In our study, two cultivars, LJ43 and BHZ, were selected to construct the F1 population; both are national tea cultivars with different advantages. LJ43 is one of the main cultivars for the product 'Longjing Tea', which has unique flavours, whereas early sprouting and high yield are features of BHZ.

In the current research, it was confirmed that the population size can affect the accuracy of genetic maps and QTL analysis, and as the population size increases, the map is more precise [48]. In addition, more than 200 individuals is considered a sufficient quantity for constructing a genetic map [49]. However, it is difficult to construct a sufficient F1 population because the hybridization rate in tea plants is relatively low by both natural and artificial methods. To our knowledge, the population size of all published genetic maps has been from 46 to 183 in tea plants, which may be insufficient for constructing genetic maps and lead to distortion. Through massive hybridization between LJ43 and BHZ, we obtained a relatively large-scale F1 population containing 327 individuals, which provided a higher accuracy for population size.

\section{High-density genetic map and QTL mapping}

In this paper, we present the densest genetic map for a tea plant published thus far. Our map covered 
Table 4 QTLs detected in the F1 population

\begin{tabular}{|c|c|c|c|c|c|c|}
\hline Traits & QTL name & LGs & Position & Marker $^{a}$ & $\operatorname{LOD}^{\mathrm{b}}$ & $R^{2} \%^{c}$ \\
\hline \multirow[t]{4}{*}{ OPC } & qOPC03a_2015 & LG03 & 112.82 & $\mathrm{dm} 58$ & 7.4 & 20.3 \\
\hline & qOPC08a_2015 & LG08 & 14.70 & f707 & 17.24 & 42.0 \\
\hline & qOPC08b_2015 & LG08 & 33.92 & $f 1360$ & 11.8 & 29.7 \\
\hline & qOPC08c_2015 & LG08 & 58.24 & df710 & 9.87 & 27.1 \\
\hline \multirow[t]{3}{*}{ YSC } & qYSC03a_2015 & LG03 & 112.82 & dm58 & 10.75 & 14.7 \\
\hline & qYSC08a_2015 & LG08 & 14.70 & f707 & 38.94 & 42.8 \\
\hline & qYSC08b_2015 & LG08 & 33.07 & $\mathrm{f} 430$ & 28.15 & 33.5 \\
\hline \multirow[t]{5}{*}{ GC } & qGC01a_2014 & LG01 & 74.15 & F1147 & 3.82 & 9.8 \\
\hline & qGC01a_2015 & LG01 & 76.62 & df487 & 9.79 & 13.5 \\
\hline & qGC10a_2015 & LG10 & 23.95 & f369 & 6.67 & 9.4 \\
\hline & qGC10b_2015 & LG10 & 40.77 & h302 & 6.94 & 9.7 \\
\hline & qGC10c_2015 & LG10 & 59.29 & h171 & 7.37 & 10.5 \\
\hline \multirow[t]{5}{*}{ EGC } & qEGC03a_2014 & LG03 & 121.20 & h90 & 3.70 & 9.6 \\
\hline & qEGC03a_2015 & LG03 & 94.41 & f908 & 7.07 & 10.1 \\
\hline & qEGC06a_2015 & LG06 & 59.03 & f1192 & 10.56 & 14.3 \\
\hline & qEGC10a_2014 & LG10 & 43.37 & dm629 & 5.38 & 13.9 \\
\hline & qEGC10a_2015 & LG10 & 53.49 & f951 & 6.00 & 8.4 \\
\hline \multirow[t]{2}{*}{ C } & qC12a_2014 & LG12 & 0.00 & m997 & 4.34 & 15.2 \\
\hline & qC12a_2015 & LG12 & 14.67 & $\mathrm{f} 730$ & 7.03 & 9.9 \\
\hline \multirow[t]{2}{*}{ CAF } & qCAF11a_2014 & LG11 & 91.90 & $\mathrm{dm} 870$ & 4.04 & 15.7 \\
\hline & qCAF11b_2015 & LG11 & 35.34 & f983 & 5.74 & 9.2 \\
\hline \multirow[t]{5}{*}{$\mathrm{EC}$} & qEC03a_2015 & LG03 & 94.47 & h41 & 5.40 & 7.8 \\
\hline & qEC06a_2015 & LG06 & 64.25 & $\mathrm{dm} 16$ & 6.72 & 9.5 \\
\hline & qEC10a_2014 & LG10 & 39.81 & h188 & 4.11 & 10.6 \\
\hline & qEC13a_2014 & LG13 & 75.27 & h343 & 3.96 & 10.3 \\
\hline & qEC13a_2015 & LG13 & 96.74 & f1003 & 6.44 & 10.3 \\
\hline \multirow[t]{5}{*}{ EGCG } & qEGCG01a_2015 & LG01 & 110.87 & m1038 & 5.28 & 7.7 \\
\hline & qEGCG06a_2014 & LG06 & 42.35 & f654 & 4.52 & 11.5 \\
\hline & qEGCG06a_2015 & LG06 & 49.3 & df326 & 8.68 & 11.9 \\
\hline & qEGCG06b_2014 & LG06 & 64.25 & $\mathrm{dm} 16$ & 5.24 & 13.7 \\
\hline & qEGCG06b_2015 & LG06 & 58.47 & $f 661$ & 8.33 & 11.5 \\
\hline GCG & qGCG11a_2015 & LG11 & 10.32 & f1859 & 7.85 & 18.9 \\
\hline \multirow[t]{4}{*}{ ECG } & qECG06a_2014 & LG06 & 63.96 & df885 & 3.14 & 8.2 \\
\hline & qECG06b_2015 & LG06 & 45.97 & f881 & 5.29 & 7.5 \\
\hline & qECG12a_2014 & LG12 & 56.54 & m527 & 3.15 & 8.3 \\
\hline & qECG12a_2015 & LG12 & 64.6 & df676 & 6.13 & 9.1 \\
\hline
\end{tabular}

andicates the closely linked marker in the LOD peak of QTL

${ }^{b}$ Indicates the logarithm of odds score

c Indicates the percentage of phenotypic variation explained

$1678.52 \mathrm{cM}$ with an average distance of $0.4 \mathrm{cM}$ between adjacent markers, which is the smallest average distance reported for tea plants. Furthermore, spanning the total genetic map, the gap in more than $98 \%$ of adjacent markers was less than $2 \mathrm{cM}$, indicating that most markers were equally and densely distributed in the genetic map. In addition, compared with the framework map by SSR markers in our previous study, the marker order was almost identical between the two maps, indicating that the high-density map developed here was accurate and that the gaps in the framework map were filled by 
Table 5 Information on the candidate marker/gene related to flavonoid function

\begin{tabular}{lllll}
\hline Marker & $\begin{array}{l}\text { Gene } \\
\text { name }\end{array}$ & LG & Position & Description \\
\hline $\mathrm{f707}$ & CSA000778 & LG08 & 14.70 & $\begin{array}{l}\text { Basic helix-loop-helix (bHLH) } \\
\text { domain }\end{array}$ \\
$\mathrm{f252}$ & CSA005342 & LG12 & 52.99 & Chalcone-flavanone isomerase \\
\hline
\end{tabular}

these newly SNP markers. Thus, our map will be a useful tool for marker-assisted breeding, QTL mapping of important agricultural traits and identification of functional genes.

In this study, 27 major QTLs associated with flavonoids were identified in LG01, LG03, LG06, LG08, LG10, LG11, LG12, and LG13, 7 of which were related to the traits of OPC and YSC. Of note, the major QTLs were in LG03 and LG08 because the QTLs of two traits were identified in the same 3 regions with a high LOD value (7.4 to 38.94). Among them, 3 major QTLs of YSC were reported in our previous study [26]. The OPC is a key factor affecting the colour of young buds. Thus, there are key functional genes for the synthesis of anthocyanin in the abovementioned regions. Moreover, qEC03a, qEGC03a, qEGC10a and qECG12a were identified in this study and were located at the same position as qEC3_2010, qEGC3_2010, qEGC10_2010 and qECG $12 \_2010$ in a previous study [18]. Additionally, 13 other QTLs related to catechin components were found to be novel, and no similar position was identified in previous studies. Notably, 6 novel QTLs (qEGC06a, qEC06a, qEGCG06a, qEGCG06b, qECG 06a, qECG06b) of 4 traits were clustered in the near or overlapping region in LG06, and 5 QTLs (qEC10a, qEGC10a, qGC10a, qGC10b, qGC10c) were clustered in LG10. These QTL clusters mean that this region may have some functional genes controlling the metabolism of various catechin components. In addition, a QTL of CAF was located for the first time, and two major QTLs were identified in LG11 explaining 9.2 to $15.7 \%$ of the phenotypic variation.

\section{Two candidate marker/genes in LG08 and LG12}

Based on QTL analysis and function annotation, two markers, f707 and f252, were selected as the markers most likely to be involved in the metabolism of flavonoids. Firstly, anthocyanins are produced by a specific branch of the flavonoid pathway. The marker f707/CSA000778 gene has a domain that is predicted to encode transcription factor bHLH, which plays important roles in various developmental processes of eukaryotes, and one of the function is to participate in anthocyanin biosynthesis. bHLH family members have a basic helix-loop-helix domain that
Lc was the first plant bHLH protein to be isolated[50]. In the Arabidopsis, anthocyanin biosynthesis genes are divided into two subgroups: early biosynthesis genes (EBGs) are activated by co-activator independent R2R3-MYB transcription factors, whereas late biosynthesis genes (LBGs) require an MBW complex which is a ternary complex to activity the anthocyanin biosynthesis genes[51]. The ternary complex consists of MYB, bHLH, and WD40, which is a key transcription factor in the regulation of anthocyanin synthesis in most species. Then, f707 was directly mapped at the peak LOD value of qOPC08a and qYSC08a, which are QTLs for anthocyanin-related traits. Thus, we assume that the encoded protein (bHLH) of the marker f707/ CSA000778 gene may be a part of MYB-bHLHWD40, and this candidate marker/gene is worthy of further investigation. The marker f252/CSA005342 gene was annotated as having the function of chalcone-flavanone isomerase $(\mathrm{CHI})$, which is one of the key enzymes that regulates flavonoid biosynthesis. As we know, chalcone synthase (CHS) and $\mathrm{CHI}$ work on the early stages of flavonoid biosynthesis. In the tea plant, coumaroyl-CoA and malonyl-CoA were catalysed to chalcone by CHS. And then, chalcone was further transformed into flavanone by $\mathrm{CHI}$, which can derivate to almost all of the flavonoid compounds $[52,53]$. Thus, this candidate gene should be investigated in more detail for verification in a further study.

\section{Conclusions}

We genotyped a large number of F1 individuals (327) from tea plants $(\mathrm{LJ} 43 \times \mathrm{BHZ})$ using $2 \mathrm{~b}-\mathrm{RAD}$ sequencing. A high-density genetic map of the tea plant was constructed with 4217 loci (427 published SSRs and 3800 newly SNPs). To our knowledge, it is the highest density genetic map that was constructed for a tea plant, with an average interval of $0.40 \mathrm{cM}$ between adjacent markers in this study. Furthermore, this map was used to identify the QTLs of ten traits related to flavonoids in the tea plant. Finally, 27 QTLs distributed in 8 LGs were identified. In addition, two markers were selected as candidate genes that may be involved in flavonoid metabolism. The present study provides important information for gene discovery, marker-assisted selection breeding and map-based cloning for functional genes related to the flavonoid content in tea plants.

\section{Additional files}

Additional file 1: Table S1. The statistics of sequencing results (the adaptors consisted of recognition base and overhang). (XLSX 34 kb) Additional file 2: Table S2. The sequencing depth of 2b-RAD. (XLSX $31427 \mathrm{~kb})$ 
Additional file 3: Table S3. The information on SNP loci (the ref sequences of $33 \mathrm{bp}$ tag had been removed 3' sticky end). (XLSX $1788 \mathrm{~kb}$ ) Additional file 4: Table S4. The genotyping in the F1 population. (XLSX $15834 \mathrm{~kb}$ )

Additional file 5: Table S5. The details of female and male genetic maps. (XLSX $10 \mathrm{~kb}$ )

Additional file 6: Figure S1. The genetic map of the F1 population (the Co-dominate markers were named: ' $h$ '- ': hkxhk', 'f'- ': Imxll', 'm'- ': nnxnp', the dominate markers were named: 'df'- ': Imxll', 'dm'- ': nnxnp'). (ZIP 203345 kb)

Additional file 7: Table S8. The position information of SNP markers in the linkage group. (XLSX $170 \mathrm{~kb}$ )

Additional file 8: Figure S2. A comparison of current and previous genetic maps (map 1 is our SNP genetic map, map 2 quoted from previous study). (ZIP $210362 \mathrm{~kb}$ )

Additional file 9: Figure S3. The QTLs of ten flavonoid-related traits. (TIF $939 \mathrm{~kb}$ )

Additional file 10: Table S6. The results of alignment between the genetic map and the reference genome. (XLSX $121 \mathrm{~kb}$ )

Additional file 11: Figure S4. The annotated markers in the genetic map. (TIF 1089 kb)

Additional file 12: Table S7. The marker annotation of the genetic map. (XLSX 26 kb)

\section{Abbreviations}

C: Catechin; CAF: Caffeine content; cM: CentiMorgans; EC: Epicatechin; ECG: Epicatechin gallate; EGC: Epigallocatechin; EGCG: Epigallocatechin gallate; GC: Gallocatechin; GCG: Gallocatechin gallate; LOD: Logarithm of odds; OPC: Anthocyanin content; QTL: Quantitative trait locus; SNP: Single nucleotide polymorphism; SSR: Simple sequence repeat; YSC: Young shoot colour

\section{Acknowledgements}

First of all, I would like to extend my sincere gratitude to my supervisor, Hao Cheng, for his instructive advices and useful suggestions on my paper. I am deeply grateful of he helps in the completion of this paper. I am also deeply indebted to all the other tutors for their directly and indirectly help to me. special thanks should go to my friends and classmates who have put considerable time and effort into their comments on the draft. Finally, I am indebted to my parents and girlfriend for their continuous support and encouragement.

\section{Funding}

This subject was supported by Earmarked Fund for China Agriculture Research System (CARS-19); the Key Scientific and Technological Project for New Variety of Tea Plant Breeding of Zhejiang Province (2016C02053); Central Public-interest Scientific Institution Basal Research Fund (NO. 1610212018004).

\section{Availability of data and materials}

The plant materials were growing in our resource nursery which can provide to other interested researchers anytime. The $2 \mathrm{~b}$-RAD sequencing data of our obtained had been submitted to the database of NCBI SRA (accession: SRP149282). The reference genome database that was used for alignment in our study came from the NCBI SRA Database under accession PRJNA381277. The genome assembly, gene prediction, gene functional annotations, and transcriptomic data of reference genome is available at http:// www.plantkingdomgdb.com/tea_tree/.

\section{Authors' contributions}

$X L Y$ carried out the experiment, collected and organized the data and wrote the manuscript. TLQ provided the phenotype data of catechin component. WK assisted in design experiments and data analysis. SJJ assisted in the experiment. WLY and $\mathrm{CH}$ are Co-corresponding authors and directed the study. All authors read and approved the final manuscript.

\section{Ethics approval and consent to participate}

Not applicable

\section{Consent for publication}

Not applicable

\section{Competing interests}

The authors declare that they have no competing interests.

\section{Publisher's Note}

Springer Nature remains neutral with regard to jurisdictional claims in published maps and institutional affiliations.

\section{Author details}

${ }^{1}$ National Centre for Tea Improvement, Tea Research Institute, Chinese Academy of Agricultural Sciences, Hangzhou 310008, China. ${ }^{2}$ College of Horticulture and Forestry Science, Huazhong Agricultural University, Wuhan 430070, China. ${ }^{3}$ College of Horticulture, Sichuan Agricultural University, Chengdu 611130, China.

Received: 7 January 2018 Accepted: 20 November 2018

Published online: 22 December 2018

\section{References}

1. Lai X, Schnable JC. Harnessing the Potential of the Tea Tree Genome. Mol Plant. 2017;10(6):788-90.

2. Marventano S, Salomone F, Godos J, Pluchinotta F, Del Rio D, Mistretta A, Grosso G. Coffee and tea consumption in relation with non-alcoholic fatty liver and metabolic syndrome: A systematic review and meta-analysis of observational studies. Clin Nutr. 2016;35(6):1269-81.

3. Meegahakumbura M, Wambulwa MC, Thapa K, Li M, Möller M, Xu J, Yang J, Liu B, Ranjitkar S, Liu J. Indications for three independent domestication events for the tea plant (Camellia sinensis (L.) O. Kuntze) and new insights into the origin of tea germplasm in China and India revealed by nuclear microsatellites. PloS one. 2016;11(5):e0155369.

4. Gunathilaka RD, Smart JC, Fleming CM. The impact of changing climate on perennial crops: the case of tea production in Sri Lanka. Climatic Change. 2017;140(3-4):577-92.

5. Zhang C-C, Wang L-Y, Wei K, Wu L-Y, Li H-L, Zhang F, Cheng H, Ni D-J. Transcriptome analysis reveals self-incompatibility in the tea plant (Camellia sinensis) might be under gametophytic control. BMC genomics. 2016;17(1):359.

6. Tao A, Huang L, Wu G, Afshar RK, Qi J, Xu J, Fang P, Lin L, Zhang L, Lin P. High-density genetic map construction and QTLs identification for plant height in white jute (Corchorus capsularis L.) using specific locus amplified fragment (SLAF) sequencing. BMC genomics. 2017;18(1):355.

7. Jia J, Zhao S, Kong X, Li Y, Zhao G, He W, Appels R, Pfeifer M, Tao Y, Zhang $X$. Aegilops tauschii draft genome sequence reveals a gene repertoire for wheat adaptation. Nature. 2013;496(7443):91.

8. Tan LQ, Wang LY, Wei K, Zhang CC, Wu LY, Qi GN, Cheng H, Zhang Q, Cui QM, Liang JB. Floral transcriptome sequencing for SSR marker development and linkage map construction in the tea plant (Camellia sinensis). Plos One. 2013;8(11):e81611.

9. Scholten OE, van Kaauwen MP, Shahin A, Hendrickx PM, Keizer LP, Burger K, van Heusden AW, van der Linden CG, Vosman B. SNP-markers in Allium species to facilitate introgression breeding in onion. BMC Plant Biol. 2016; 16(1):187.

10. Helmkampf M, Wolfgruber TK, Bellinger MR, Paudel R, Kantar MB, Miyasaka SC, Kimball H, Brown A, Veillet A, Read A. Phylogenetic relationships, breeding implications, and cultivation history of Hawaiian taro (Colocasia esculenta) through genome-wide SNP genotyping. J Hered. 2017;109(3):272-82.

11. Zhang $X$, Zhang H, Li L, Lan H, Ren Z, Liu D, Wu L, Liu H, Jaqueth J, Li B. Characterizing the population structure and genetic diversity of maize breeding germplasm in Southwest China using genome-wide SNP markers. BMC genomics. 2016;17(1):697.

12. Fukuda S, Ishimoto K, Sato S, Terakami S, Hiehata N, Yamamoto T. A highdensity genetic linkage map of bronze loquat based on SSR and RAPD markers. Tree Genetics Genomes. 2016;12(4):80.

13. Song Q, Jenkins J, Jia G, Hyten DL, Pantalone V, Jackson SA, Schmutz J, Cregan PB. Construction of high resolution genetic linkage maps to improve the soybean genome sequence assembly Glyma1.01. BMC Genomics. 2016;17(1):33.

14. Liu N, Li M, Hu X, Ma Q, Mu Y, Tan Z, Xia Q, Zhang G, Nian H. Construction of high-density genetic map and QTL mapping of yield-related and two 
quality traits in soybean RILs population by RAD-sequencing. BMC Genomics. 2017;18(1):466.

15. Jiang Z, Wang H, Michal JJ, Zhou X, Liu B, Woods LCS, Fuchs RA. Genome wide sampling sequencing for SNP genotyping: methods, challenges and future development. Int J Biol Sci. 2016;12(1):100.

16. Wang S, Meyer E, McKay JK, Matz MV. 2b-RAD: a simple and flexible method for genome-wide genotyping. Nat methods. 2012;9(8):808-10.

17. Chang Y, Oh EU, Lee MS, Kim HB, Moon D-G, Song KJ. Construction of a genetic linkage map based on RAPD, AFLP, and SSR markers for tea plant (Camellia sinensis). Euphytica. 2017;213(8):190.

18. Ma J-Q, Yao M-Z, Ma C-L, Wang X-C, Jin J-Q, Wang X-M, Chen L. Construction of a SSR-based genetic map and identification of QTLs for catechins content in tea plant (Camellia sinensis). PloS one. 2014;9(3):e93131.

19. Ma J-Q, Huang L, Ma C-L, Jin J-Q, Li C-F, Wang R-K, Zheng H-K, Yao M-Z, Chen L. Large-scale SNP discovery and genotyping for constructing a highdensity genetic map of tea plant using specific-locus amplified fragment sequencing (SLAF-seq). PloS one. 2015;10(6):e0128798.

20. Chen D, Milacic V, Chen MS, Wan SB, Lam WH, Huo C, Landis-Piwowar KR, Cui QC, Wali A, Chan TH. Tea polyphenols, their biological effects and potential molecular targets. Histol Histopathol. 2008;23(4):487.

21. Dixon RA, Pasinetti GM. Flavonoids and isoflavonoids: from plant biology to agriculture and neuroscience. Plant Physiol. 2010;154(2):453-7.

22. Higdon JV, Frei B. Tea catechins and polyphenols: health effects, metabolism, and antioxidant functions; 2003.

23. Han ZX, Rana MM, Liu GF, Gao MJ, Li DX, Wu FG, Li XB, Wan XC, Wei S. Green tea flavour determinants and their changes over manufacturing processes. Food Chem. 2016;212:739-48.

24. Feng L, Gao MJ, Hou RY, Hu XY, Zhang L, Wan XC, Wei S. Determination of quality constituents in the young leaves of albino tea cultivars. Food Chem. 2014;155(11):98-104.

25. Li C-F, Zhu Y, Yu Y, Zhao Q-Y, Wang S-J, Wang X-C, Yao M-Z, Luo D, Li X, Chen L. Global transcriptome and gene regulation network for secondary metabolite biosynthesis of tea plant (Camellia sinensis). BMC Genomics. 2015;16(1):560

26. Tan L-Q, Wang L-Y, Xu L-Y, Wu L-Y, Peng M, Zhang C-C, Wei K, Bai P-X, Li H$\mathrm{L}$, Cheng H. SSR-based genetic mapping and QTL analysis for timing of spring bud flush, young shoot color, and mature leaf size in tea plant (Camellia sinensis). Tree Genetics Genomes. 2016;12(3):52.

27. Jiao W, Fu X, Dou J, Li H, Su H, Mao J, Yu Q, Zhang L, Hu X, Huang X. Highresolution linkage and quantitative trait locus mapping aided by genome survey sequencing: building up an integrative genomic framework for a bivalve mollusc. DNA Res. 2014;21(1):85-101.

28. Fu X, Dou J, Mao J, Su H, Jiao W, Zhang L, Hu X, Huang X, Wang S, Bao Z. RADtyping: an integrated package for accurate de novo codominant and dominant RAD genotyping in mapping populations. PloS One. 2013;8(11): e79960

29. Li R, Yu C, Li Y, Lam T-W, Yiu S-M, Kristiansen K, Wang J. SOAP2: an improved ultrafast tool for short read alignment. Bioinformatics. 2009;25(15): 1966-7.

30. Tan L-Q, Wang L-Y, Xu L-Y, Wu L-Y, Peng M, Zhang C-C, Wei K, Bai P-X, Li H$L$, Cheng H. SSR-based genetic mapping and QTL analysis for timing of spring bud flush, young shoot color, and mature leaf size in tea plant (Camellia sinensis). Tree Genetics Genomes. 2016;12(3):1-13.

31. Grattapaglia D, Sederoff R. Genetic linkage maps of Eucalyptus grandis and Eucalyptus urophylla using a pseudo-testcross: mapping strategy and RAPD markers. Genetics. 1994;137(4):1121-37.

32. Stam P. Construction of integrated genetic linkage maps by means of a new computer package: Join Map. Plant J. 1993;3(5):739-44.

33. Voorrips R. MapChart: software for the graphical presentation of linkage maps and QTLs. J Hered. 2002;93(1):77-8.

34. Wu Y, Close TJ, Lonardi S. Accurate construction of consensus genetic maps via integer linear programming. IEEE/ACM Trans Comput Biol Bioinform. 2011:8(2):381-94.

35. Fishman L, Kelly AJ, Morgan E, Willis JH. A genetic map in the Mimulus guttatus species complex reveals transmission ratio distortion due to heterospecific interactions. Genetics. 2001;159(4):1701-16.

36. Chakravarti A, Lasher LK, Reefer JE. A maximum likelihood method for estimating genome length using genetic linkage data. Genetics. 1991;128(1): 175-82.

37. Van Ooijen J. MapQTL $\left\llcorner^{-5}\right.$. Software for the mapping of quantitative trait loci in experimental populations: Plant \& Animal Genome XII Conference; 2004
38. Cui F, Zhao C, Ding A, Li J, Wang L, Li X, Bao Y, Li J, Wang H. Construction of an integrative linkage map and QTL mapping of grain yield-related traits using three related wheat RIL populations. Tagtheor Appl Genet Und Angewandte Genetik. 2014;127(3):659.

39. Xia EH, Zhang HB, Sheng J, Li K, Zhang QJ, Kim C, Zhang Y, Liu Y, Zhu T, Li W. The Tea Tree Genome Provides Insights into Tea Flavor and Independent Evolution of Caffeine Biosynthesis. Mol Plant. 2017;10(6):866-77.

40. Xu W, Grain D, Bobet S, Gourrierec J, Le E, Thévenin J, Kelemen Z, Lepiniec L, Dubos C. Complexity and robustness of the flavonoid transcriptional regulatory network revealed by comprehensive analyses of MYB-bHLH-WDR complexes and their targets in Arabidopsis seed. New Phytol. 2014;202(1):132.

41. Zhao X, Yuan Z, Feng L, Fang Y. Cloning and expression of anthocyanin biosynthetic genes in red and white pomegranate. J Plant Res. 2015; 128(4):687.

42. Charrier B, Coronado C, Kondorosi A, Ratet P. Molecular characterization and expression of alfalfa ( Medicago sativa L.) flavanone-3-hydroxylase and dihydroflavonol-4-reductase encoding genes. Plant Mol Biol. 1995;29(4):773-86.

43. Catchen J, Hohenlohe PA, Bassham S, Amores A, Cresko WA. Stacks: an analysis tool set for population genomics. Mol Ecol. 2013;22(11):3124.

44. Wang S, Lv J, Dou J, Lu Q, Zhang L, Bao Z. Genotyping by Sequencing and Data Analysis: RAD and 2b-RAD Sequencing: Principles and Methods: Wiley, Ltd; 2017.

45. Dou J, Li X, Fu Q, Jiao W, Li Y, Li T, Wang Y, Hu X, Wang S, Bao Z. Evaluation of the $2 b-R A D$ method for genomic selection in scallop breeding. Sci Rep. 2016;6(9):19244

46. Cui Z, Hui M, Liu Y, Song C, Li X, Li Y, Liu L, Shi G, Wang S, Li F. High-density linkage mapping aided by transcriptomics documents ZW sex determination system in the Chinese mitten crab Eriocheir sinensis. Hered. 2015;115(3):206.

47. Henery ML, Moran GF, Wallis IR, Foley WJ. Identification of quantitative trait loci influencing foliar concentrations of terpenes and formylated phloroglucinol compounds in Eucalyptus nitens. New Phytol. 2007;176(1): 82-95.

48. Li H, Hearne S, Bänziger M, Li Z, Wang J. Statistical properties of QTL linkage mapping in biparental genetic populations. Hered. 2010;105(3):257-67.

49. Ferreira A, MFd S, Cruz CD. Estimating the effects of population size and type on the accuracy of genetic maps. Genet Mol Biol. 2006;29(1):187-92.

50. Ramsay NA, Glover BJ. MYB-bHLH-WD40 protein complex and the evolution of cellular diversity. Trends Plant Sci. 2005;10(2):63-70.

51. Petroni K, Tonelli $\mathrm{C}$. Recent advances on the regulation of anthocyanin synthesis in reproductive organs. Plant Sci An Int J Exp Plant Biol. 2011; 181(3):219-29.

52. PA P, IS A, V K DT, D D CG, S M GF, TC F. Flavonoid biosynthesis in the tea plant Camellia sinensis: properties of enzymes of the prominent epicatechin and catechin pathways. Arch Biochem Biophys. 2004;431 (1):22-30.

53. Saslowsky D, Winkel-Shirley B. Localization of flavonoid enzymes in Arabidopsis roots. Plant J Cell Mol Biol. 2010;27(1):37-48.

\section{Ready to submit your research? Choose BMC and benefit from:}

- fast, convenient online submission

- thorough peer review by experienced researchers in your field

- rapid publication on acceptance

- support for research data, including large and complex data types

- gold Open Access which fosters wider collaboration and increased citations

- maximum visibility for your research: over $100 \mathrm{M}$ website views per year

At BMC, research is always in progress.

Learn more biomedcentral.com/submissions 